\title{
Resultados da correção cirúrgica de esotropias de grande ângulo, em portadores de baixa de acuidade visual unilateral
}

\author{
Results of surgical treatment of large angle esotropia in patients with low visual acuity
}

Jorge Antonio Meireles-Teixeira

Rosana Pires da Cunha

Tomás Scalamandré Mendonça
Endereço para correspondência: $\mathrm{R}$. Afonso Brás - 804/112. Vila Nova Conceição. São Paulo (SP) CEP: 04511-001.e-mail: jorgemeireles@bol.com.br

\begin{tabular}{|c|}
\hline RESUMO \\
\hline $\begin{array}{l}\text { Objetivo: Avaliar os resultados cirúrgicos de esotropias de grande } \\
\text { ângulo (no mínimo } 60 \text { dioptrias prismáticas - dp), associadas à baixa de } \\
\text { acuidade visual (BAV) unilateral, cuja cirurgia foi planejada com o } \\
\text { intuito de não se operar o olho de melhor visão. } \\
\text { Casuística e Métodos: Foram selecionados } 17 \text { casos de esotropias } \\
\text { não-acomodativas, associadas à BAV (AV } \leq 0,4 \text { no olho não-fixador, } \\
\text { com a melhor correção) e sem tratamento cirúrgico prévio. Foi consi- } \\
\text { derado bom resultado desvio pós-operatório de no máximo } 10 \text { dp, com } \\
\text { rotações binoculares de até }-2 \text { de reto medial e }+2 \text { de reto lateral, } \\
\text { regular XT / ET entre } 10 \text { e } 15 \text { dp, inclusive, ou rotações de } 3 \text { e ruim } \\
\text { se tinha XT / ET }>15 \text { dp ou rotações de } \pm 4 \text {. } \\
\text { Resultados: } 13 \text { ( } 76,4 \%) \text { tinham AV de conta-dedos no olho não- } \\
\text { fixador, } 2 \text { (11,7\%) atingiam } 0,1 \text { e outros } 2 \text { (11,7\%) } 0,4 \text {. Em } 3 \text { havia } \\
\text { alta miopia (equivalente esférico } \geq-6,00 \text { dioptrias esféricas) em } \\
\text { ambos os olhos. Entre os } 17 \text { pacientes, } 12 \text { (70,5\%) obtiveram bom } \\
\text { resultado cirúrgico, } 3 \text { (17,6\%) foram regulares e } 2 \text { (11,7\%) ruins. } \\
\text { Conclusão: A cirurgia de estrabismo sob anestesia tópica mostrou } \\
\text { ser eficaz e segura nestes casos especiais de BAV em um dos olhos, } \\
\text { sendo que na maioria das vezes consegue-se não operar o olho de } \\
\text { melhor visão; o que a nosso ver, só se tornou possível pelo uso da } \\
\text { anestesia tópica. }\end{array}$ \\
\hline
\end{tabular}

Palavras-chave: Esotropia grande; Cirurgia monocular; Anestesia tópica.

\section{INTRODUÇÃO}

Nas esotropias de ângulo grande, não existe consenso quanto ao tipo de abordagem cirúrgica mais adequada, visto serem desvios de correção menos previsível.

Tradicionalmente, o tipo de abordagem cirúrgica eleita para desvios de ângulo maior que 60 dioptrias prismáticas (dp), tem sido a cirurgia seletiva - operação nos quatro músculos retos horizontais. No entanto, alguns estudos surgiram contrapondo-se a este tipo de procedimento, em prol da cirurgia uniforme - cirurgia em apenas dois músculos, bilateralmente ou da cirurgia monocular ${ }^{1-4}$.

As vantagens do tratamento das esotropias de grande ângulo com operação em poucos músculos (menor tempo cirúrgico e preservação de alguns músculos ainda intocados para o caso de reoperação), mostraram-se superiores às suas supostas desvantagens (ineficácia para corrigir desvios tão grandes e o surgimento de limitações importantes na movimentação dos 
olhos). Alguns autores, analisando casos de esotropias congênitas concluíram ser a cirurgia uniforme bastante viável para a correção de esotropias de grande ângulo, tanto do ponto de vista da correção do desvio em si, quanto da limitação da movimentação ocular que poderia advir ${ }^{2,5,6}$. Talvez a cirurgia em poucos músculos possa equilibrar melhor o sistema oculomotor já bastante prejudicado destes pacientes ${ }^{7}$.

No Setor de Motilidade Ocular Extrínseca do Departamento de Oftalmologia da Escola Paulista de Medicina, em casos de baixa de acuidade visual (BAV) unilateral, é planejada a cirurgia tentando-se evitar operar o olho fixador, portanto com poucos músculos, usando-se anestesia tópica. Neste estudo analisamos a cirurgia feita em tais pacientes.

\section{CASUÍSTICA E MÉTODOS}

Foram selecionados os pacientes operados no ambulatório de cirurgia tópica de estrabismo da Escola Paulista de Medicina, que apresentassem desvio convergente não acomodativo, de no mínimo 60dp e com BAV associada (máximo de 0,4 de acuidade visual no pior olho, com a melhor correção), nunca antes operados.

Foram feitas no mínimo duas medidas pré-operatórias do desvio, com correção óptica total se fosse o caso e com intervalo médio de 1 mês entre as duas. O tempo de acompanhamento pós-operatório de todos os pacientes foi de 6 meses. Os que tinham menos de 6 meses de seguimento foram excluídos. Também foram eliminados os pacientes que tivessem desvio vertical associado à esotropia.

A medida do desvio foi feita pelo método de Krimsky ou pelo teste de cobertura alternada com prisma, sempre que possível. Procedeu-se a avaliação da movimentação ocular através da observação das rotações binoculares, usando uma escala de hipo e hiperfunções de -4 à +4 .

Ao todo foram revisados 309 prontuários entre janeiro/96 a fevereiro/99, dos quais selecionaram-se 20 pacientes. Todos os pacientes foram operados sob anestesia tópica e foi feito ajuste per-operatório da cirurgia. Nos casos nos quais não foi possível corrigir todo o desvio operando-se somente um olho (3 pacientes), optou-se por fazer também um recuo do reto medial do olho fixador e estes pacientes não foram considerados para a análise dos dados, visto terem fugido do objetivo do estudo, de se poupar o olho fixador de cirurgia.

Considerou-se como bom resultado os casos cujo desvio após a cirurgia ficou entre ortotropia e 10 dioptrias prismáticas (dp) de eso ou exotropia e cuja rotação binocular ficou com até no máximo 2 de hipofunção (-) do reto medial (RM) ou hiperfunção (+) do reto lateral (RL). O resultado cirúrgico entre 10 a $15 \mathrm{dp}$, inclusive, de eso ou exotropia e/ou rotação binocular de \pm 3 foi considerado regular. Desvios pós-cirúrgicos superiores a $15 \mathrm{dp}$ e/ ou rotação binocular de \pm 4 foram classificados como ruins.

Os casos que necessitaram de reoperação foram considerados ruins e para eles, foi tomada como base para a avaliação, a última medida feita antes da segunda cirurgia.
Dos 17 pacientes incluídos no estudo, a grande maioria, 15 $(88,2 \%)$, eram do sexo feminino e apenas $2(11,7 \%)$ do masculino. Predominaram também os adultos jovens (20 a 30 anos), com 9 casos (52,9\%) do total, seguido dos adultos (40 a 59 anos), que representaram 4 pessoas $(23,5 \%)$. Por fim, tivemos os adolescentes ( 10 a 19 anos), 3 ao todo $(17,6 \%)$ e os idosos ( $\geq 60$ anos), com apenas 1 paciente $(5,8 \%)$.

Entre todos os pacientes, $13(76,4 \%)$ tinham acuidade visual de no máximo conta-dedos (CD) no pior olho; 2 $(11,7 \%)$ conseguiam enxergar até 0,1 com o olho de menor visão e outros $2(11,7 \%$ ) atingiam 0,4 (Tabela 1). Três pacientes também apresentavam alta miopia (equivalente esférico $\geq-6,00$ dioptrias esféricas) em ambos os olhos.

\section{RESULTADOS}

O maior número de indivíduos, $10(58,8 \%)$ apresentou desvio entre 60 e $70 \mathrm{dp}$, dos quais 9 (90\%) obtiveram bons resultados, contra apenas $1(10 \%)$ com resultado ruim (Gráficos 1 e 2). Neste primeiro grupo, em todos os pacientes a cirurgia realizada foi recuo - ressecção $(\mathrm{Rc}-\mathrm{Rs})$ dos retos horizontais do olho de pior visão; sendo que o recuo médio foi de 7,4mm (5,0 a 9,0mm), medidos a partir da inserção do músculo reto medial e a ressecção média de $7,95 \mathrm{~mm}(6,0$ a $10,0 \mathrm{~mm}$ ). O único caso com resultado desfavorável estava associado à alta miopia, tendo ficado com esotropia residual de $25 \mathrm{dp}$ (ruim).

Dos pacientes com desvio maior que $70 \mathrm{dp}, 7$ (41,1\%) no total, $3(42,8 \%)$ destes obtiveram bons resultados, $3(42,8 \%)$ regulares e $1(14,2 \%)$ ruim (Gráficos 1 e 2). A cirurgia em 3 músculos ( Rc - Rs no pior olho, mais recuo do RM do olho fixador) só foi feita em 3 pacientes, tendo sido 1 com bom resultado, 1 regular e outro ruim. Porém estes pacientes não foram incluídos na casuística, por terem fugido ao objetivo do trabalho.

Considerando-se todos os 17 pacientes, 12 (70,5\%) foram classificados como bons resultados, 3 (17,6\%) como regulares e $2(11,7 \%)$ como ruins (Gráficos 1 e 2$)$. Um caso ruim estava associado à alta miopia.

Com relação às rotações binoculares, tivemos apenas dois casos com hipofunção de -2 de RM recuado. Analisando-se o RL, observa-se que houve um caso de -3 do RL ressecado (Tabela 1), no entanto, este paciente tinha -7 antes da cirurgia (paresia de VI nervo) e um desvio exagerado (150dp).

\section{DISCUSSÃO}

Como todos os pacientes analisados tinham BAV em um dos olhos, tentou-se sempre fazer cirurgia monocular, ainda que com recuos e ressecções teoricamente exagerados. $\mathrm{O}$ objetivo desta abordagem, para os nossos pacientes, seria o de lhes corrigir o desvio sem se operar o olho com que efetivamente enxergam. Porém, os estudos que avaliam a 


\begin{tabular}{|c|c|c|c|c|}
\hline \multicolumn{5}{|c|}{ Tabela 1. Dados sobre os pacientes } \\
\hline Idade / Sexo & $\begin{array}{l}\text { Acuidade } \\
\text { Visual* }^{*}\end{array}$ & $\begin{array}{l}\text { Desvio Pré e } \\
\text { Pós-Op. }\end{array}$ & Cirurgia** & $\begin{array}{l}\text { Rotações Binoc. } \\
\text { Pós-Cirúrgicas*** }\end{array}$ \\
\hline $44 \mathrm{~F}$ & $0,9 / \mathrm{CD} 4 \mathrm{~m}$ & ET60 / XT 10 & $\mathrm{Rc7,0} / \mathrm{Rs} 8,0$ & -1RM / 0 RL \\
\hline $15 \mathrm{~F}$ & $0,6 / \mathrm{CD} 20 \mathrm{~cm}$ & ET60 / ET10 & Rc9,0 / Rs10,0 & $-1 R M /-1 R L$ \\
\hline $43 \mathrm{~F}$ & $0,6 / C D 1 \mathrm{~m}$ & ET150 / ET5 & Rc9,0 / Rs12,0 & ? / -3 RL \\
\hline $14 \mathrm{~F}$ & $1,0 / \mathrm{CD} 20 \mathrm{~cm}$ & ET95 / ORTO & $\mathrm{Rc7}, 0$ / Rs8,0 & $-1 R M /-1 R L$ \\
\hline $16 \mathrm{~F}$ & $1,0 / \mathrm{SPL}$ & ET60 / ORTO & Rc6,0 / Rs8,0 & $-1 R M /-1 R L$ \\
\hline $22 \mathrm{~F}$ & $1,0 / \mathrm{CD} 50 \mathrm{~cm}$ & ET60 / ORTO & $\mathrm{RC7,0} / \mathrm{Rs} 8,0$ & -1RM / 0 RL \\
\hline $39 \mathrm{~F}$ & $0,4 / C D 2 m$ & ET60 / ORTO & $\mathrm{RC} 8,0$ / Rs8,0 & $-1 \mathrm{RM} / 0 \mathrm{RL}$ \\
\hline $63 \mathrm{~F}$ & $0,5 / \mathrm{MM}$ & ET95 / ET25 & Rc8,0 / Rs7,0 & $0 \mathrm{RM} /$-1RL \\
\hline $22 \mathrm{~F}$ & $1,0 / 0,4$ & ET60 / ET8 & Rc8,0 / Rs7,5 & $-1 R M /-1 R L$ \\
\hline $28 \mathrm{~F}$ & $1,0 / C D 3 m$ & ET65 / ET25 & $\mathrm{RC} 8,0$ / Rs8,0 & $-1 R M /-2 R L$ \\
\hline $21 \mathrm{~F}$ & $0,6 / 0,1$ & ET70 / ORTO & $\mathrm{RC7,0} / \mathrm{Rs} 6,0$ & $0 \mathrm{RM} /-1 \mathrm{RL}$ \\
\hline $34 \mathrm{~F}$ & $1,0 / 0,1$ & ET75 / ET10 & Rc8,0 / Rs9,0 & $0 \mathrm{RM} / 0 \mathrm{RL}$ \\
\hline $20 M$ & $0,9 / \mathrm{CD} 1 \mathrm{~m}$ & ET75 / XT15 & Rc8,0 / Rs9,0 & -2 RM / 0 RL \\
\hline $24 \mathrm{~F}$ & $0,3 / \mathrm{CD} 2 \mathrm{~m}$ & ET60 / ORTO & Rc9,0 / Rs8,0 & 0 a $-1 \mathrm{RM} / 0 \mathrm{RL}$ \\
\hline $40 \mathrm{M}$ & $1,0 / 0,4$ & ET75 / ET5 & Rc9,0 / Rs10,0 & 0 a $-1 \mathrm{RM} /-1 \mathrm{RL}$ \\
\hline $31 \mathrm{~F}$ & $1,0 / \mathrm{CD} 30 \mathrm{~cm}$ & ET90 / ET15 & Rc8,0 / Rs9,0 & -2 RM / 0 RL \\
\hline $43 \mathrm{~F}$ & $1,0 / \mathrm{CD} 30 \mathrm{~cm}$ & ET60 / ET4 & Rc5,0 / Rs8,0 & -1RM / 0 RL \\
\hline \multicolumn{5}{|c|}{$\begin{array}{l}\text { *A primeira anotação corresponde ao olho fixador, independentemente da lateralidade; } \\
\text { ** Rc: recuo (sempre de RM - Reto Medial); Rs: ressecção (sempre de RL - Reto Lateral); } \\
\text { ** Os valores das rotações binoculares estão dispostos correspondendo respectivamente às anotações da cirurgia feita e só estão assinaladas as rotações dos } \\
\text { músculos que foram operados; } \\
\text { M: masculino; F: feminino; CD: conta-dedos; MM: movimento de mãos; SPL: sem percepção luminosa; ET: esotropia; XT: exotropia; ORTO: ortotropia }\end{array}$} \\
\hline
\end{tabular}

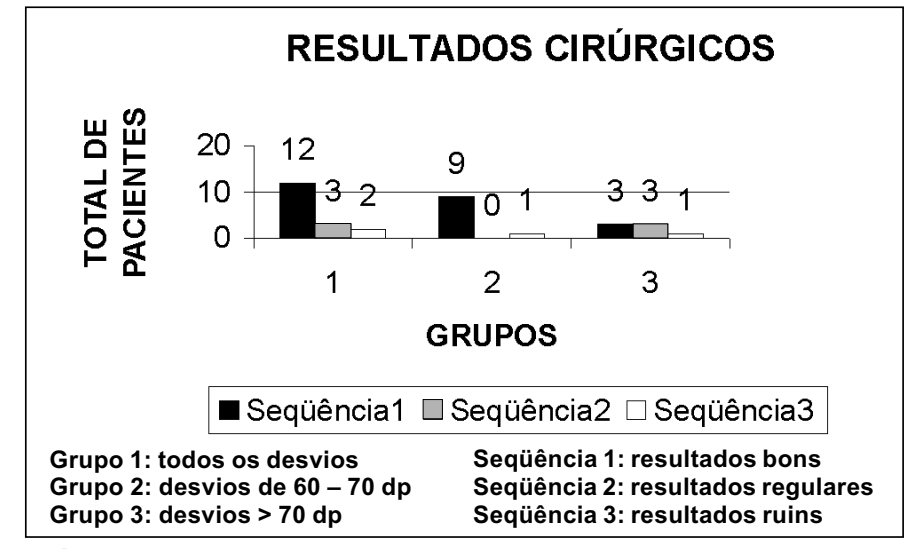

Gráfico 1

\section{DESVIO X CORRÉÇÃO CIRÚRGICA}

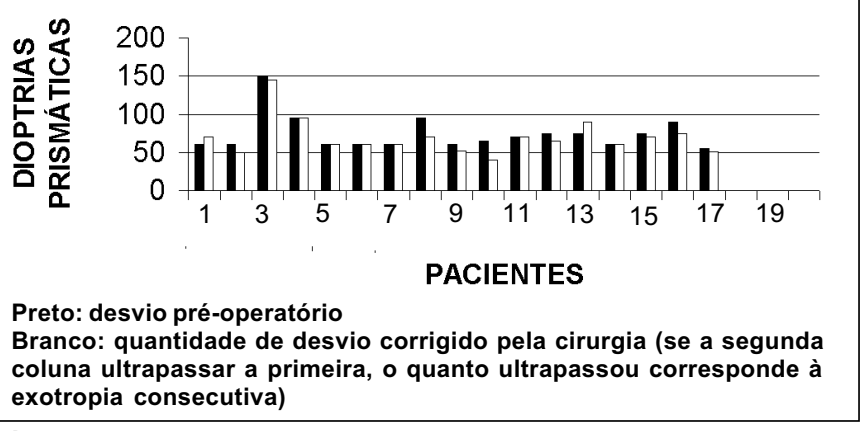

Gráfico 2 correção de esotropias com poucos músculos, o fazem em pacientes com esotropia congênita, corrigida com duplo recuo de retos mediais em torno de 7,0 a $7,5 \mathrm{~mm}^{5,2}$. Neste estudo, onde em todos os pacientes foi realizado também ressecção, em geral ampla, isto só foi factível graças ao uso da anestesia tópica, por permitir a avaliação per-operatória do efeito da cirurgia. Só assim, pode-se ter segurança que recuos e ressecções acentuados, em poucos músculos, não trariam severas alterações da movimentação ocular, a despeito do bom alinhamento conseguido. E nos casos que, para se obter bom resultado, causar-se-ia limitações muito grandes das rotações, optou-se por operar um músculo do outro olho, neste caso o RM. Por exemplo: se se observasse, através do exame das rotações, uma limitação de -3 no RM recuado no pior olho, decidia-se, no mesmo tempo cirúrgico, diminuir o recuo feito e operar também o RM do olho fixador (ao todo isto ocorreu em três pacientes, cujos dados não foram analisados neste estudo).

A cirurgia de estrabismo sob anestesia tópica mostrou-se ser um procedimento eficaz para a correção de grandes desvios, nestes casos particulares de BAV severa em um olho e, na maioria das vezes, consegue-se bom resultado poupando-se o olho de melhor visão de uma cirurgia.

Apesar do nosso acompanhamento ter se limitado a 6 meses, os pacientes em alta ambulatorial são orientados a retornar ao serviço, caso haja alguma piora. Isto nos faz crer que a maioria deve ter se mantido com a correção satisfatória, uma vez que não voltaram.

Seria interessante comparar-se os nossos resultados com os de outros pacientes também com BAV unilateral, com grande ângulo de desvio e que tivessem sido operados 
segundo a abordagem seletiva (operando-se os 4 retos horizontais). É sabido que o Rc - Rs costuma ser mais estável, a longo prazo, que o duplo recuo isoladamente ${ }^{8}$. Em todos os nossos pacientes foi realizado Rc - Rs, podendo-se esperar que os nossos resultados a longo prazo sejam parecidos aos da cirurgia seletiva, feita numa amostra semelhante.

\section{SUMMARY}

Purpose: To evaluate the surgical outcome of large angle esotropia (minimum of 60 prism diopters - pd) associated with unilateral low visual acuity (LVA) in which the surgical strategy was applied in order to operate only the non-fixating eye.

Patients and Methods: We selected 17 patients with nonaccommodative esotropia, LVA (VA 0,4 in the nonfixating eye with the better visual correction) and no previous treatment. Patients were classified as good result if they had a postoperative deviation of maximally $10 \mathrm{pd}$ with binocular rotations of up to -2 of medial rectus and +2 of lateral rectus. Exotropia/esotropia between 10 and 15 pd or binocular rotations of \pm 3 were considered as a regular outcome. Deviations greater than 15 pd or binocular rotations of \pm 4 were included regarded as poor results.

Results: Thirteen (76.4\%) patients had counts fingers VA in the non-fixating eye, 2 (11.7\%) achieved 0.1 and another two had 0.4. Three of them were also highly myopic (spherical equivalent of -6.00 diopters) in both eyes. Among the 17 patients, 12 (70.5\%) had a good surgical outcome, 3 (17.6\%) were regular and 2 (11.7\%) poor.

Conclusions: Strabismus surgery under topical anesthesia proved to be reliable in these special cases of unilateral LVA and often one can avoid surgery of the fixating eye by using this method.

Keywords: Large esotropias; Monocular surgery; Topical anesthesia.

\section{REFERÊNCIAS BIBLIOGRÁFICAS}

1. Scott WE, Reese PD, Hirsh CR, Flabetich CA. Surgery for large - angle congenital esotropia: two vs. three and four horizontal muscles. Arch Ophthalmol 1986;104:374-7.

2. Weakley DR, Parks MM. Results from $7 \mathrm{~mm}$ bilateral recessions of the medial rectus muscles for congenital esotropia. Ophthalmic Surg 1990;21(12):827-30.

3. Richler M, Barsoum-Homsy M. Bimedial rectus recession measured from the limbus for congenital esotropia. Can J Ophthalmol 1989;24(7):304-7.

4. Lucci L, Goldchmit M, Souza-Dias C. Influência dos chamados "fatores de supercorreção" sobre o resultado da cirurgia para correção de esotropia de grande ângulo. Arq Bras Oftal 1997;60(3):286-9.

5. Nelson LB et al. Surgical management of large angle congenital esotropia. Br J Ophthalmol 1987;71:380-3.

6. Szmyd SM, Nelson LB, Calhoun JH, Spratt C. Large bimedial rectus recession in congenital esotropia. Br J Ophthalmol 1985;69:271-4.

7. Prieto-Diaz J. Large bilateral medial rectus recession in early esotropia with bilateral limitation of abduction. J Pediatr Ophthalmol Strabismus 1980;17:101-5.

8. Von Noorden GK. Binocular Vision and Ocular Motility: theory and management of strabismus, 5th ed. St. Louis: Mosby 1996;537.

\title{
XXIV SIMAsp SIMPÓSIO INTERNACIONAL MOACYR ÁLVARO
}

\author{
"Oftalmologia de Resultados: Facoemulsificação, Glaucoma, \\ Refrativa e Cirurgia Ocular"
}

15 a 17 de Fevereiro de 2001

SÃO PAULO - SP

Informações: Tel./Fax: (1 1) 5575-4243

e-mail:ceo@oftalmo.epm.br 